\title{
Time-Frequency Entropy Analysis of Arc Signal in Non-Stationary Submerged Arc Welding
}

\author{
Kuanfang $\mathrm{He}^{1}$, Siwen Xiao ${ }^{1}$, Jigang $\mathrm{Wu}^{1}$, Guanbin Wang ${ }^{2}$ \\ ${ }^{1}$ Hunan Provincial Key Laboratory of Health Maintenance for Mechanical Equipment, \\ Hunan University of Science and Technology, Xiangtan, China \\ ${ }^{2}$ Engineering Research Center of Advanced Mine Equipment, Ministry of Education, \\ Hunan University of Science and Technology, Xiangtan, China \\ E-mail:hkf791113@163.com,xswxssw888@shou.com,jgwuhust@gmail.com,jxxwgb@126.com \\ Received December 24, 2010; revised January 4, 2011; accepted January 20, 2011
}

\begin{abstract}
The use of time-frequency entropy to quantitatively assess the stability of submerged arc welding process considering the distribution features of arc energy is reported in this paper. Time-frequency entropy is employed to calculate and analyze the stationary current signals, non-stationary current and voltage signals in the submerged arc welding process. It is obtained that time-frequency entropy of arc signal can be used as arc stability judgment criteria of submerged arc welding. Experimental results are provided to confirm the effectiveness of this approach.
\end{abstract}

Keywords: Non-Stationary Signal, Submerged Arc Welding, Time-Frequency Entropy, Stability

\section{Introduction}

The submerged arc welding has been widely used in welding production because of its high effection [1]. But it is hard to realize online inspection of voltaic arc and molten pool state by detection methods of infrared and video, because the arc load burn under the welding fluid and influenced by various uncertainties, and the processes of arc stability, drop transfer and weld formation are changed. Although strongly nonlinear, time-varying load of arc, the changes of arc made energy signals is reflected in time and frequency domain in welding process, so it a significant to achieve online detection and study arc stability and welding quality by sensed current or voltage signals in welding process [2]. Welding current or voltage is a no-stationary signal of submerged arc welding. The time-frequency analysis is the useful method to research no-stationary signal [3]. So it's no doubt that time-frequency analysis and calculation of arc current and voltage signals of submerged arc welding is a good method to judge stability of arc and welding quality by introducing information entropy into time-frequency distribution $[4,5]$. In order to quantitatively describe the degree for the energy time-frequency distribution of different operation state of a machine, information entropy theory as a diagnosis criterion has been introduced into the time-frequency distribution by some researchers, and good effect has been obtained in machinery fault diagnosis [6,7]. Therefore, time-frequency analysis method is introduced to analyze the collected arc signal in different conditions of welding process and calculate corresponding energy entropy in this paper. The relationship between arc stability and energy entropy of submerged arc welding is explored to obtain new knowledge of arc characteristic of submerged arc welding, even to get more accurate numerical evaluation of arc stability. It is also provided that a more adequate guidance for welding parameters choice.

\section{Time-Frequency Entropy Theory and Algorithm}

There are some time-frequency analysis methods such as window fourier transformation, continuous wavelet transformation, wigner-ville distribution and hilbert-huang transformation. The time-frequency window in window fourier transformation is immovable, so it can't meet the frequency and time resolution at the same time. In the process of wavelet transformation, the choice of wavelet basis has a great impact on analysis result with poor adaptation to the whole signal. Wigner-ville has cross-term disturbance, so it is against to the subsequent time-frequency entropy calculation. Hilbert-huang Transform is a new self-adapting time-frequency analysis method. It has higher time-frequency resolution and time-frequency 
concentration, which is especially suitable to the analysis of arc current signal. Hilbert-huang transform is introduced to conduct time-frequency analysis of arc current signal.

Hilbert-huang transformation contains empirical mode decomposition and corresponding hilbert spectrum. The signal $x(t)$ is decomposed into summation of lots of internal mode component Intrinsic Mode Function $c_{i}$ and a residual component $r_{\mathrm{n}}$ as expressed in Equation (1),

$$
x(t)=\sum_{i=1}^{n} c_{i}+r_{n}
$$

where $r_{\mathrm{n}}$ is an average trend or a constant. Neglecting the residual component $r_{n}$, Hilbert transform of every Intrinsic Mode Function $c_{i}(t)$ is performed. Equation (2) can be obtained,

$$
\widehat{c_{i}}(t)=\frac{1}{\pi} \int_{-\infty}^{\infty} \frac{c_{i}(\tau)}{t-\tau} d \tau
$$

The analytic signal $Z_{i}(t)$ is constructed by Equation (2),

$$
z_{i}(t)=c_{i}(t)+j \widehat{c_{i}}(t)=a_{i}(t) e^{j \phi_{i}(t)}
$$

Where the amplitude function $a_{i}(t)$ and phase function $\phi_{i}(t)$ are obtained as Equations (4) and (5) respectively,

$$
\begin{aligned}
a_{i}(t) & =\sqrt{c^{2}{ }_{i}(t)+\widehat{c^{2}{ }_{i}(t)}} \\
\phi_{i}(t) & =\arctan \frac{\widehat{c_{i}}(t)}{c_{i}(t)}
\end{aligned}
$$

The instantaneous frequency $f_{i}(t)$ can be further obtained by Equation (5) as follows,

$$
f_{i}(t)=\frac{1}{2 \pi} \omega_{i}(t)=\frac{1}{2 \pi} \times \frac{d \phi_{i}(t)}{d t}
$$

In this way, the following Equation (7) can be obtained,

$$
x(t)=R P \sum_{i=1}^{n} a_{i}(t) e^{j \phi_{i}(t)}=R P \sum_{i=1}^{n} a_{i}(t) e^{j \int \omega_{i}(t) d t}
$$

where $R P$ is real part. Equation (7) is defined as Hilbert spectrum and is written as Equation (8),

$$
H(\omega, t)=R P \sum_{I=1}^{N} a_{i}(t) e^{j \int \omega_{i}(t) d t}
$$

Equation (8) describes the accurate changing law of time and frequency of signal amplitude in the whole frequency ranges. Signal amplitude is expressed as function of time and instantaneous frequency in the three dimensions space. It also can be expressed as contour line of time-frequency plane.

The method of information entropy introduced into time-frequency analysis is to divide time-frequency plane into $N$ equal area blocks. Each block energy is sup- posed as $W_{i}(i=1,2, \cdots, N)$, the total energy of the time-frequency plane is $A$. Each block energy is normalized by $q_{i}=W_{i} / A$, thus $\sum_{i=1}^{N} q_{i}=1$, which corresponds with initial normalization condition of information entropy calculation. Based on the formula of information entropy, formula of time-frequency entropy $s(q)$ based on Hilbert-huang transformation is written as Equation (9),

$$
s(q)=-\sum_{i=1}^{N} q_{i} \operatorname{In} q_{i}
$$

According to the basic property of information entropy, the more uniform is the $q_{i}$ distribution, the larger is the value of time-frequency entropy $s(q)$, while the less uniform is the $q_{i}$ distribution, and the smaller is the value of time-frequency entropy.

\section{Time-Frequency Entropy Calculation of Arc Signal}

Experiments have been done by submerged arc welding machine ME1000. The material of work piece is low carbon steel with slab thickness of $20 \mathrm{~mm}$, the welding wire trademark is H08A with diameter of $4.0 \mathrm{~mm}$, and welding flux is HJ431. Current and voltage signals are collected by sensor SML1500E/S60 and data acquisition card PCI-1718HGU in the experiments. Under the above experimental condition, experimental program is done as follows: a) Under the given voltage of $38 \mathrm{~V}$, current amplitude of 450A, welding wire extension of $22 \mathrm{~mm}$, welding speed of $0.6 \mathrm{~m} / \mathrm{min}$, the welding parameter unchanged in the welding process. b) Under the given voltage of $38 \mathrm{~V}$, welding wire extension of $22 \mathrm{~mm}$, welding speed of $0.6 \mathrm{~m} / \mathrm{min}$, current amplitude changed from $450 \mathrm{~A}$ to 550A. c) Under the given current amplitude of 450A, welding wire extension of $22 \mathrm{~mm}$, welding speed of 0.6 $\mathrm{m} / \mathrm{min}$, the welding voltage changed in the welding process. Technology experiment are done by the above three experiments and record the arc signal in welding process. The frequency of sampling is $20 \mathrm{kHz}$, sample time is 30 $\mathrm{s}$ in each welding process. Time-frequency entropy calculation is done by 200000 points from the whole acquisition data. The waveform of welding arc current and voltage are shown in Figures 1-3.

Each 5000 collected data of currents and voltage is analyzed respectively with Hilbert-huang transformation, 100 time-frequency entropy value $s_{a}$ in each duty cycle is obtained. $s_{\text {aavg }}$ is the average of $s_{a}$. The calculation result of time-frequency entropy $s_{a}$ is shown in Figure 4.

From Figure 1, it can be seen that welding process are stable. It is indicated that physical parameter of current, voltage and inputting energy are unchanged. Figure $\mathbf{2}$ is 


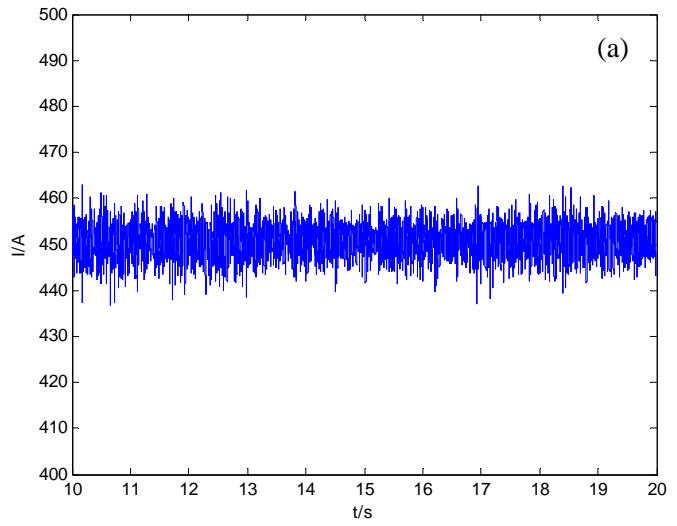

(a) Current signals

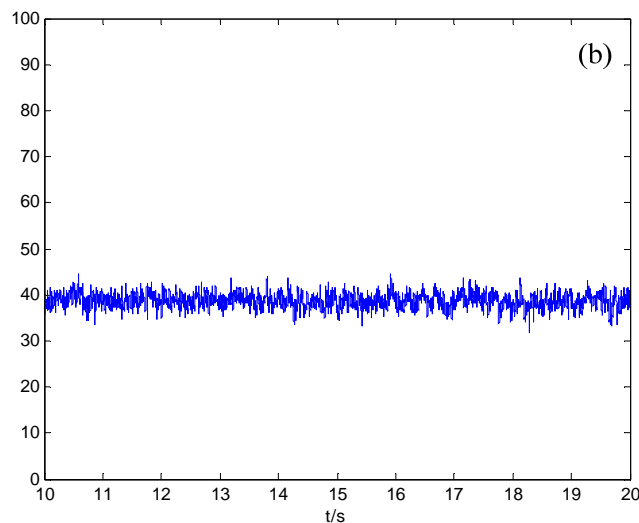

(b) Voltage signals

Figure 1. the arc signals the arc signals under experimental program (a).

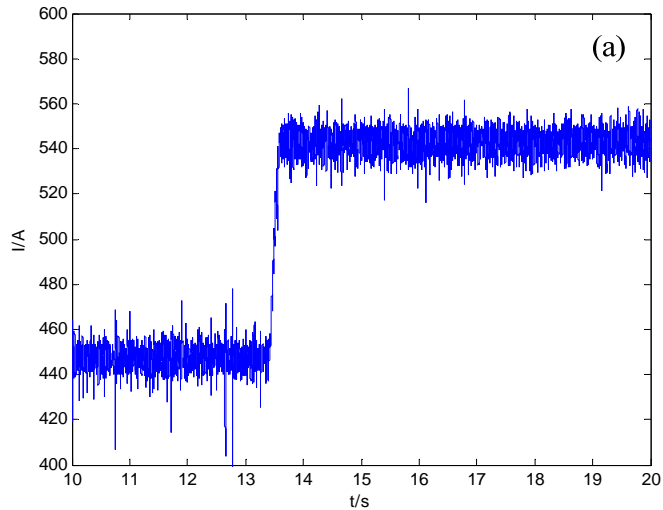

(a) Current signals

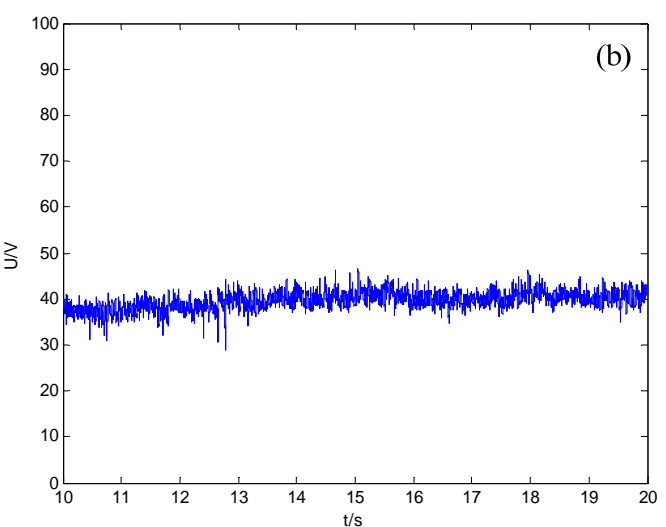

(b) Voltage signals

Figure 2. The arc signals under experimental program (b).

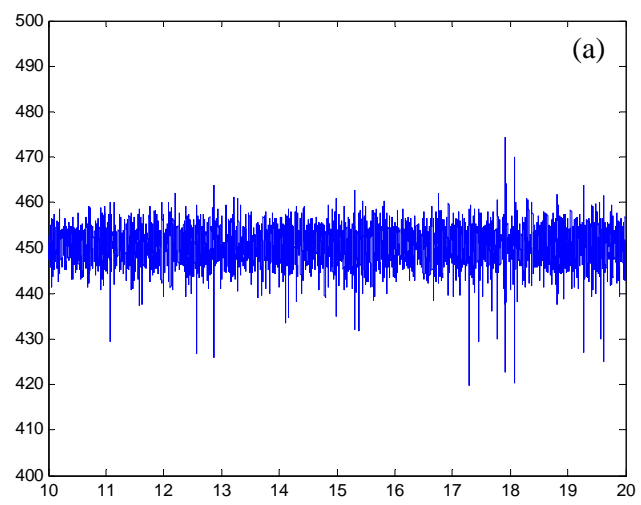

(a) Current signals

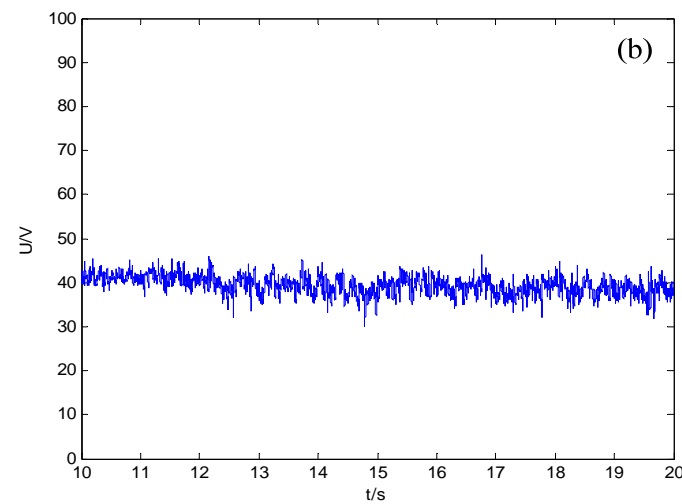

(b) Voltage signals

Figure 3. The arc signals under experimental program (c).

the current and voltage signal of suddenly changed arc, there are two steps in the current waveform, and each platform is very stable, the before platform is near the current $450 \mathrm{~A}$, the after platform is near the current 550A, the change of the current occurs in a very short time.
Figure 3 shows the arc current is always stable, but arc voltage is slightly undulating, which cause the arc energy fluctuations and instability inputting of welding heat.

Table 1 shows that time-frequency entropy calculation result that is big as the arc current and voltage are more 
Table 1. Welding parameters and time-frequency entropy in different experiments.

\begin{tabular}{cccccccc}
\hline $\begin{array}{c}\text { experimental } \\
\text { program }\end{array}$ & $\begin{array}{c}\text { Wire } \\
\text { diameter } \\
/ \mathrm{mm}\end{array}$ & $\begin{array}{c}\text { Dry extension } \\
/ \mathrm{mm}\end{array}$ & Current & Voltage & Current & Voltage & Welding conditions \\
\hline a) & 4 & 22 & 0.449319 & 0.466446 & 0.00102 & 0.000997 & $\begin{array}{c}\text { Stable process and good } \\
\text { formality welding seam } \\
\text { Stable process and formality } \\
\text { welding seam changed } \\
\text { unstable process and bad } \\
\text { formality welding seam }\end{array}$ \\
\hline
\end{tabular}

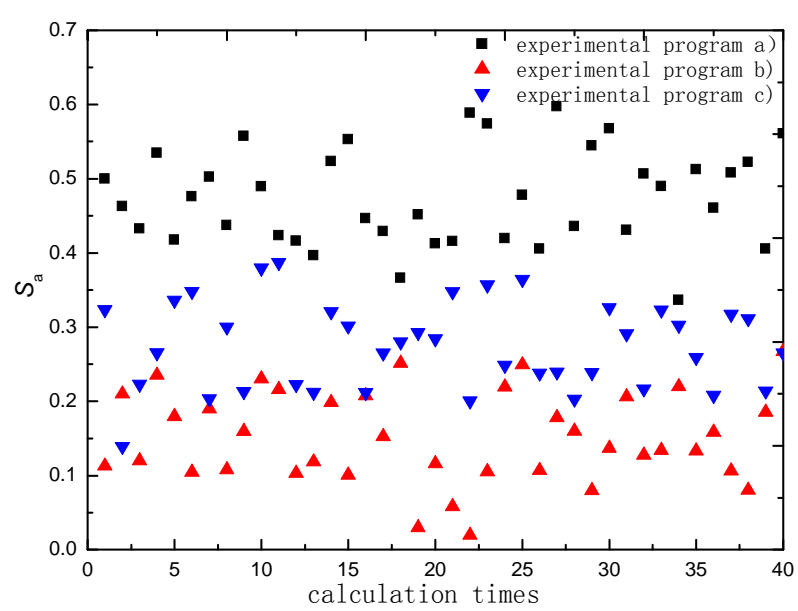

(a) Current

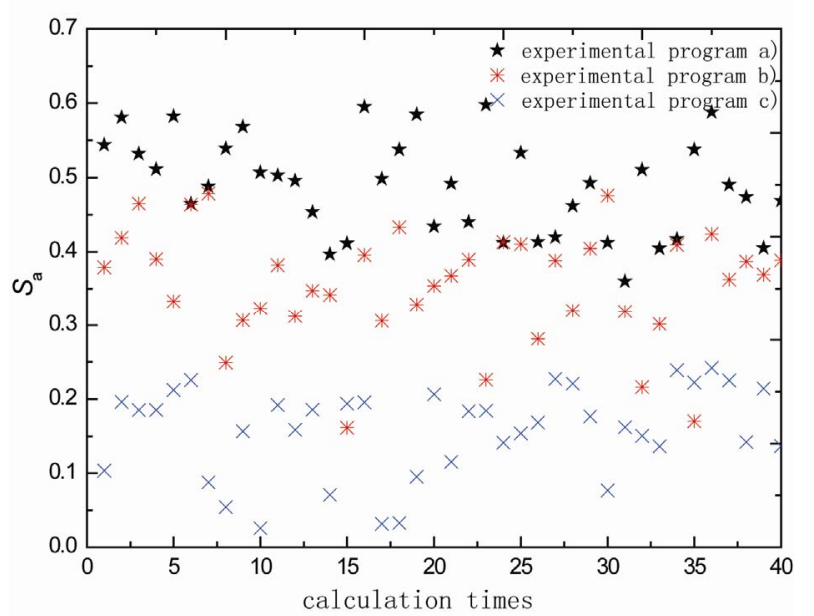

(b) Voltage

Figure 4. Time-frequency entropy .

stable. The time-frequency entropy is a measure of com plexity, so it should be large as current and voltage are regular. The time-frequency is reduced as arc current and voltage changed. It indicates that the arc current and voltage wave of submerged arc welding is a factor to affect the time-frequency entropy, and is closely related with stability of welding process. Therefore, time-frequency entropy can be used as the stability judgment criteria of submerged arc welding. Furthermore, it is seen form Figure 2 and Table 1 that the welding current can be adjusted to obtain the appropriate weld parameters width, penetration. Figure 3 and Table 1 show that the key factor of inputting weld energy is arc voltage in the conditions of unchanged current. Under certain conditions of welding current, the time-frequency entropy changes larger as arc voltage variation and lead to deterioration of the weld shape.

Figure 4 shows the fluctuations of time-frequency entropy calculation process. It also can be seen from the process of time-frequency entropy calculation that the more stability of submerged arc welding process is, the smaller of fluctuations of current and time-frequency entropy are. The standard deviation $\sigma\left(S_{\mathrm{a}}\right)$ is the numerical description of fluctuations of time-frequency entropy calculation process, the smaller is the $\sigma\left(S_{\mathrm{a}}\right)$, and the smaller is the fluctuations. Table 1 shows that standard deviation of time-frequency entropy is the smallest in experimental program a), which indicates that the fluctuations under these experimental program are smallest and most stability. It is also verified that time-frequency entropy can be used as the stability judgment criteria of submerged arc welding.

\section{Conclusions}

The calculation and analysis of time-frequency entropy under three experimental condition show that time-frequency entropy value relates with stability of submerged arc welding process. The more stable is the welding process, the larger is the time-frequency entropy. On the other hand, the more unstable is the welding process, the larger is the fluctuations of time-frequency entropy. It is verified that the larger time-frequency entropy indicates the more stable arc. This research indicates that the timefrequency entropy can be used as stability evaluation criterion for alternating current square wave submerged arc welding.

\section{Acknowledgements}

This work was supported by the National Natural Sci- 
ence Foundation of China (No. 51005073), Scientific Research Fund of Hunan Provincial Education Department (No. 10C0682), Ph. D Start Fund (No. E51088), Aid program for Science and Technology Innovative Research Team in Higher Educational Institutions of Hunan Province and Hunan Natural and Science Foundation Project (No. 10JJ8002).

\section{References}

[1] D. S. Tusek, "Mathematical Modeling of Melting Rate in Twin-Wire Welding," Journal of Materials Processing Technology, Vol. 100, No. 1-3, 2000, pp. 250-256. doi:10. 1016/S0924-0136(99)00485-9

[2] L. Yi, "Application of Joint Time-Frequency Analysis to Electrical Signals of $\mathrm{CO}_{2}$ Arc Welding," Transactions of the China Welding Institution, Vol. 28, No. 2, 2007. pp. 75-78.
[3] L. Cohen, "Time Frequency Analysis: Theory and Applications,” Prentice Hall, New Jersey, 1995.

[4] J. H. Dong, H. M. Gu and X. Zhang, "A Comparison of Time-Frequency Analysis Methods and Their Applications," Chinese Journal of Engineering Geophysics, Vol 4, No. 4, 2007, pp. 312-316.

[5] Z. Q. Tian and Y. Zhou, "The Certification of the Fundamental Properties of Comentropy,” Journal of Inner Mongolia Normal University (Natural Science Edition), Vol. 31, No. 4, 2002, pp. 347-350.

[6] J. Lin and L. S. Qu, "Application of Time-Frequency Entropy to Crack Identification,” Journal of Mechanical Transmission, Vol. 22, No. 2, 1998, pp. 37-39.

[7] D. J. Yu, W. Zhang and J. S. Cheng, “Application of Time-Frequency Entropy to Gear Fault Diagnosis Based on Emd," Journal of Vibration and Shock, Vol. 24, No. 5, 2005, pp. 26-29. 